\title{
Gerbil Experimental Organism Diagnosis
}

National Cancer Institute

\section{Source}

National Cancer Institute. Gerbil Experimental Organism Diagnosis. NCI Thesaurus. Code C136555.

A condition that is relevant to either disease states or models of disease in gerbils (Subfamily Gerbillinae.) 